\title{
Glass-like stress relaxation of a permanent gelatin network as a signature of pretransitional helix-coil fluctuations
}

Christiane Caroli, Olivier Ronsin, and Anaël Lemaître

Citation: The Journal of Chemical Physics 148, 054901 (2018); doi: 10.1063/1.5003212

View online: https://doi.org/10.1063/1.5003212

View Table of Contents: http://aip.scitation.org/toc/jcp/148/5

Published by the American Institute of Physics

\section{Articles you may be interested in}

Multiscale weighted colored graphs for protein flexibility and rigidity analysis

The Journal of Chemical Physics 148, 054103 (2018); 10.1063/1.5016562

Theory of activated glassy dynamics in randomly pinned fluids

The Journal of Chemical Physics 148, 054502 (2018); 10.1063/1.5011247

Enhanced gel formation in binary mixtures of nanocolloids with short-range attraction

The Journal of Chemical Physics 148, 044902 (2018); 10.1063/1.5007038

Contrasting two different interpretations of the dynamics in binary glass forming mixtures

The Journal of Chemical Physics 148, 054504 (2018); 10.1063/1.5012088

Markov modeling of peptide folding in the presence of protein crowders

The Journal of Chemical Physics 148, 055101 (2018); 10.1063/1.5017031

Curvature and bottlenecks control molecular transport in inverse bicontinuous cubic phases

The Journal of Chemical Physics 148, 054902 (2018); 10.1063/1.5019224

\section{AIP| The Jounal of Chemical Physics}




\title{
Glass-like stress relaxation of a permanent gelatin network as a signature of pretransitional helix-coil fluctuations
}

\author{
Christiane Caroli, ${ }^{1}$ Olivier Ronsin, ${ }^{1}$ and Anaël Lemaître ${ }^{2}$ \\ ${ }^{1}$ INSP, Université Pierre et Marie Curie-Paris 6, CNRS, UMR 7588, 4 place Jussieu, \\ 75252 Paris Cedex 05, France \\ ${ }^{2}$ NAVIER, UMR 8205, École des Ponts, IFSTTAR, CNRS, UPE, 2 allée Képler, F-77420 Marne-la-Vallée, France
}

(Received 4 September 2017; accepted 15 December 2017; published online 1 February 2018)

\begin{abstract}
The stress response of permanently crosslinked gelatin gels was recently observed to display glass-like features, namely, a stretched-exponential behavior terminated by an exponential decay, the characteristic time scales of which increase dramatically with decreasing temperature. This phenomenon is studied here using a model of flexible polymer gel network where relaxation proceeds via elementary monomer exchanges between helix and coil segments. The relaxation dynamics of a full network simulation is found to be nearly identical to that of a model of independent strands, which shows that for flexible polymer gels in the range of elastic moduli of interest, both strand contour length disorder and elastic couplings are irrelevant. We thus focus on the independent strand model and find it not only to explain the observed functional form of the stress relaxation curves but also to yield predictions that match very satisfactorily the experimental measurements of final relaxation time and total stress drop. The system under study thus constitutes a rare case where the origin of glass-like behavior can be unambiguously identified, namely, as the signature of the enhancement of helix content fluctuations when approaching from above the mean-field helix-coil transition of strands. Published by AIP Publishing. https://doi.org/10.1063/1.5003212
\end{abstract}

\section{INTRODUCTION}

In a recent experimental study, Ronsin et al. ${ }^{1}$ found the stress response of permanently crosslinked gelatin gels to present a transient stretched exponential behavior terminated by exponential decay. They showed this behavior to originate from the strain-induced helix-coil transition ${ }^{2,3}$ on single polymer network strands, yet measured time scales (of order hours) that, when fitted by an Arrhenius law, yield apparent activation energies that are incompatibly larger than the barrier limiting the elementary helix-coil transition process.

By and large, this phenomenology can be termed "glasslike." Indeed, stretched exponential relaxation (SER), possibly terminated by exponential decay, accompanied by very large time scales that cannot be rationalized as simple Arrhenius behavior, are well-known hallmarks of relaxation in deeply supercooled liquids. ${ }^{4,5}$ Although glass-like dynamics is common to various classes of disordered systems, its physical origin remains, in most cases, quite poorly understood. It is a largely open question to what extent its occurrence in different systems is coincidental and depends on systemspecific features or originates from a few general (universal) mechanisms.

A major issue in the case of glass-formers is that relaxation proceeds via rearrangements of disordered atomic clusters with ill-defined boundaries which reshuffle the amorphous structure. As elementary relaxation events cannot be clearly isolated and modeled, existing propositions for the origin of SER have had to resort to assumptions about, e.g., a hierarchically constrained dynamics or the topology of traps in phase space that cannot be easily validated. ${ }^{6-8}$

In this context, it is of interest to identify situations where glass-like behavior can be attributed to simple mechanisms and fully analyzed. Such is the case for the gel network of Ref. 1, where relaxation has been shown to proceed via well-defined elementary transformations (monomer exchange between helix and coil segments) which, thanks to irreversible covalent cross-linking, occur at a fixed network topology. This gelatin gel, hence, lends itself to modeling under controlled assumptions in view of analyzing the origin of its glass-like dynamics.

In the context of structural glasses, an open issue is the possible influence of long-range elastic couplings on relaxation. In the gel network of Ref. 1, this effect is present since any exchange of a monomer between coil and helix domains induces a variation of the tension on the implicated strand and therefore causes the whole network to deform in order to recover mechanical balance. These strains bias later events, hence introducing long-range elastic couplings. Such couplings are neglected by construction in the rubberlike models of independent chains commonly used to study the mechanical response of gels. To test their relevance in our case, we construct a schematic model, where the gel is represented by a full network of a fixed topology. Disorder is introduced via a distribution of strand contour lengths. Each strand presents one helical and one coil domain, the respective equilibrium fractions of which depend on the strand end-to-end distance. ${ }^{2}$ The elementary dynamical event consists in the thermally activated exchange of a monomer 
between coil and helix domains followed by global mechanical equilibration.

In order to probe the importance of elastic couplings, we compare the relaxation dynamics for this model with that for a model of independent, uncoupled, strands, following thermal quenches from a hot (zero helix fraction) state. This comparison is performed at a high cross-link density corresponding to a gel elastic modulus beyond the relevant experimental range, i.e., in a situation where elastic effects are deliberately overestimated. We then find that even in the presence of significant strand contour length disorder, both the network and uncoupled chain models yield nearly identical dynamical responses, which entails that elastic coupling effects are negligible in the whole experimental range. This result, as we will discuss, can be assigned to the nearly Gaussian elasticity of coil segments.

It follows that the relaxation of our system is governed by the evolution of the distribution of helix fractions for an ensemble of independent chains. The model defines a "transition point" above (respectively, below) which the minimum of the free-energy of a strand corresponds to a zero (respectively, finite) helix segment length. ${ }^{2}$ We study in detail, at various distances from the transition, the stress relaxation following a mechanical quench and show that our model is able to match the experimental data. On this basis, we conclude that the experimental range lies near, but above, the transition point and that the glass-like slowing down at decreasing temperatures is caused by the broadening of the distribution of helix segment lengths upon approaching the transition from above. In this sense, the glass-dynamics of the gelatin gel of Ref. 1 can be fully interpreted as a pretransitional slowing down effect.

\section{PROBING THE RELEVANCE OF ELASTIC COUPLINGS}

In this section, we study a network model of gel so as to explicitly take into account long-range elastic couplings. As in Ref. 9, our model consists in a periodic 2D network with a fixed triangular topology, where the nodes represent the permanent cross-links and the links the polymer strands that rotate freely at the nodes. The system free-energy $\mathcal{F}=\mathcal{F}^{\mathrm{ch}}+\mathcal{F}^{F l}$ adds two terms, accounting for the free-energy of individual strands in an ideal solvent and for excluded volume effects. More precisely, the "chain free-energy" reads

$$
\mathcal{F}^{\mathrm{ch}}=\sum_{\{i j\}} F_{i j},
$$

where the sum runs over all strands $\{i j\}$. Each strand $i j$, which connects nodes $i$ and $j$, contains $N_{i j}$ monomers. The structural disorder characteristic of gel networks is introduced by considering that the strand contour lengths, i.e., $N_{i j}$ 's, are distributed. Strands are represented as proposed by Kutter and Terentjev ${ }^{2}$ who discuss at length the relevance of their model to the case of gelatin gels. Among the $N_{i j}$ monomers of each strand, $n_{i j}$ form a single rigid helix segment adjacent to one node, assumed to be aligned with the end-to-end vector $\underline{r}_{i j}=\underline{r}_{j}-\underline{r}_{i}$; the $N_{i j}$ $-n_{i j}$ remaining monomers form a single coil. This approximation neglects, in particular, the entropy associated with the possibility to form multiple helix domains, which appears reasonable in view of the smallness of strand lengths - which lie typically in the range of a few ten nanometers. The free-energy of strands is based on the Kutter and Terentjev expression, ${ }^{2}$ where we replace the Gaussian form of the coil free-energy by the 2D freely jointed chain approximation derived in Ref. 9 that accounts for chain stiffening at high stretch levels. The free-energy of strand $i j$ reads

$$
F_{i j}=k T \frac{a\left(N_{i j}-n_{i j}\right)}{2 \ell_{p}} w\left(\frac{r_{i j}-\gamma a n_{i j}}{a\left(N_{i j}-n_{i j}\right)}\right)+\Delta f n_{i j}
$$

with $a$ and $\ell_{p}$ the monomer and persistence lengths, and $\gamma$ a factor accounting for the shortening of the effective polymer length caused by the winding of the helix segment; $\Delta f$ is the (temperature-dependent) free-energy difference per monomer between the helix and coil configurations. The function $w$, derived in Ref. 9, reads

$$
w(x)=\frac{1}{2}\left(x^{2}-\log \left(1-x^{2}\right)\right) .
$$

It reduces to the ideal chain expression in the small stretch, $x \ll 1$ limit.

Finally, the "Flory free-energy" reads

$$
\mathcal{F}^{\mathrm{Fl}}=\frac{k_{B} T}{2}(1-2 \chi) a^{2} \sum_{\{i j k\}} c_{i j k}^{2} A_{i j k},
$$

where the sum runs over all triangles. Here, $\chi$ is the 2D Flory parameter, $A_{i j k}$ is the area of triangle $i j k$, and

$$
c_{i j k}=\frac{1}{2} \frac{N_{i j}+N_{j k}+N_{i k}}{A_{i j k}} .
$$

The evolution of the system proceeds by a series of thermally activated events during which a single monomer is added to/removed from the helix segment of a strand. Here we are concerned with a situation, akin to that of supercooled liquids, ${ }^{10}$ where the relaxation time $\tau$ is considerably larger than acoustic delays over reasonable sample sizes. Hence, we consider that each event consists in a transition between two mechanically equilibrated configurations.

The activation process, the cis-trans isomerization of a peptide bond, is characterized by an Arrhenius time $\tau_{0}=v_{0}^{-1} \exp \left[E_{0} /(k T)\right]$ of order $10 \mathrm{~s}$ at $25^{\circ} \mathrm{C}^{11,12}$ The barrier passage time itself (controlled by hydrodynamic damping ${ }^{13}$ ) lies in the nanosecond range and hence is much smaller than the Rouse time (of order $10^{-5} \mathrm{~s}$ ) of the polymer strand on which it takes place. It follows that activation can be considered to take place at the fixed cross-link positions set by mechanical equilibrium conditions in the initial state. The total energy change associated with the $n_{i j} \rightarrow n_{i j} \pm 1$ isomerization process hence reads

$$
\Delta \mathcal{F}_{i j}^{ \pm}=F_{i j}\left(n_{i j} \pm 1 ; r_{i j}\right)-F_{i j}\left(n_{i j} ; r_{i j}\right) .
$$

The corresponding barrier is estimated as

$$
\mathcal{E}_{i j}^{ \pm}=E_{0}+\frac{1}{2} \Delta \mathcal{F}_{i j}^{ \pm}
$$

which yields an associated transition rate

$$
R_{i j}^{ \pm}=v_{0} \exp \left[-\frac{\mathcal{E}_{i j}^{ \pm}}{k T}\right]=\tau_{0}^{-1} \exp \left[-\frac{\Delta \mathcal{F}_{i j}^{ \pm}}{2 k T}\right] .
$$


The time evolution of the system is simulated using kinetic Monte Carlo. Each simulation step is defined by the following operations: the rates of all possible transitions are calculated in an initial, mechanically equilibrated configuration; the time of occurrence, location (strand $i j)$, and nature $\left(n_{i j}\right.$ $\rightarrow n_{i j} \pm 1$ ) of the next isomerization event are then drawn randomly from the distributions that realize the above-defined rate process; then, mechanical balance is recovered by energy minimization.

We use $\gamma=0.4,{ }^{2} 1-2 \chi \simeq 2 \times 10^{-2},{ }^{14}$ and $\ell_{p}=7 a,{ }^{15}$ values that are appropriate to gelatin. The mesh size of the triangular network is taken to be $\xi=30 a$, and the average number of monomers per strand as $N=100$. As announced in the Introduction, these values correspond to gels much stiffer than in experiments and are chosen to artificially amplify elastic coupling effects. We examine two cases:

- "homogeneous networks," where all strands have the same monomer number $N$;

- "disordered networks," where the monomer numbers of strands are uncorrelated and set randomly to either of the two equiprobable values $N_{i j}=N \pm \Delta$, with $\Delta=50$.

In our model, temperature dependence is entirely encoded in the dimensionless parameter:

$$
\alpha \equiv \Delta f / k T .
$$

The experiments of Ref. 1 are performed at temperatures slightly above the helix-coil transition of the free polymer chains, where $\Delta f=0$. In this narrow range, $\alpha \simeq \beta\left(T-T_{0}\right)$ rises roughly linearly with temperature.

To characterize relaxation towards thermal equilibrium in our model, we study, for different values of $\alpha$, the evolution of the space-averaged helix fraction $\phi_{h}$ starting from the $\phi_{h}=0$ (helix-free, very high-temperature) configuration. Data are obtained using a network of 480 nodes. Our $\alpha$ values are chosen so that the equilibrium helix fraction remains noticeably smaller than 1 , since this is expected to be the case in experiments, as deduced by Ronsin et al. ${ }^{1}$ on the basis of their data.

Strikingly, the relaxation dynamics of both "homogeneous" and "disordered" networks, displayed in Fig. 1, are nearly identical. This weak effect of disorder on the dynamics is very surprising a priori, given that we have investigated a case where contour length fluctuations are large $(\Delta / N$ $=50 \%$ ). This we think can be understood by recalling that as we analyzed in Ref. 9, mechanical balance mitigates the effect of disorder by systematically reducing the fluctuations of stretch ratios as compared with those of contour lengths. Now, the thermodynamic force driving the formation of helices, $\partial F_{i j} / \partial n_{i j}$, is only a function of the stretch ratio of coil segments, $x_{i j}=\left(r_{i j}-\gamma a n_{i j}\right) / a\left(N_{i j}-n_{i j}\right)$, and hence is not directly sensitive to the fluctuations of $N_{i j}$ itself. Of course, we expect that the fluctuations of $x_{i j}$ 's increase with their helix content, hence that disorder effects become more significant at a high average helix fraction. This is indeed the trend observed on the top curves of Fig. 1.

At all temperatures, $\phi_{h}$ is seen to converge towards equilibrium values that, as expected, increase with decreasing

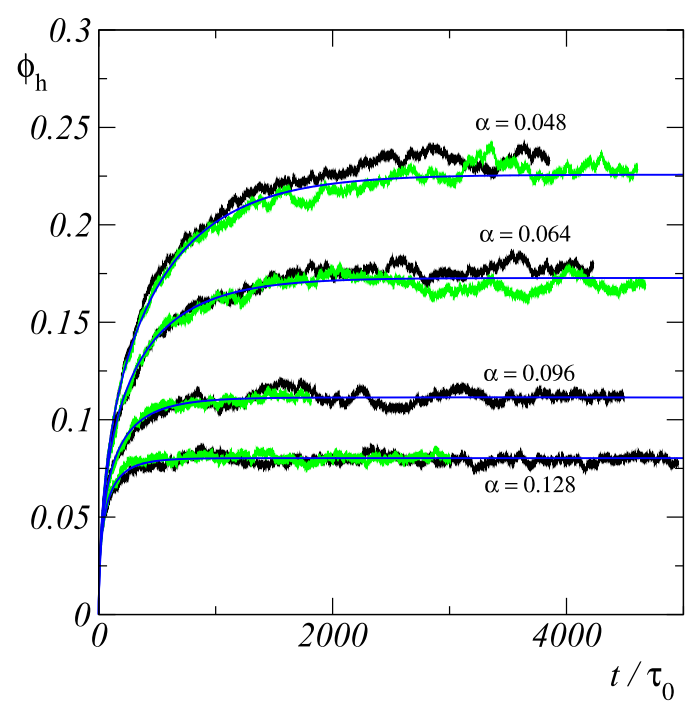

FIG. 1. The helix fraction $\phi_{h}$ vs $t / \tau_{0}$ after a quench from the high- $T$ zerohelix state for: our homogeneous (black) and disordered (green) networks, and our model of independent 2D chains (blue).

$\alpha$, i.e., decreasing $T$. Noticeably, the reduced time of convergence towards the equilibrium plateau (a rough estimate of the relaxation time) grows very rapidly, from hundreds to several thousands, with decreasing temperatures. Since times are reported in units of $\tau_{0}$, this entails that relaxation slows down considerably faster than the elementary activation process-a central feature of the experimental behavior.

The question now is to identify the physical origin of this slowing down and in particular to test the possible role of elastic couplings. In view of the weakness of disorder effects on the regime of our interest, we concentrate from now on the "homogeneous" problem and compare its relaxation dynamics with that of a model of independent chains which all have the monomer number $N=100$ and a common end-to-end distance equal to the network mesh size $\xi=30 a$. The latter problem can be formulated in terms of the master equation for the distribution $P_{n}$ of the helix monomer number $n$ on a chain,

$$
\frac{\partial P_{n}}{\partial t}=R_{n-1}^{+} P_{n-1}+R_{n+1}^{-} P_{n+1}-\left(R_{n}^{+}+R_{n}^{-}\right) P_{n},
$$

where in accordance with Eqs. (6) and (8), the rates are given by

$$
\begin{aligned}
R_{n}^{ \pm} & =\tau_{0}^{-1} \exp \left[-\frac{\Delta \mathcal{F}_{n}^{ \pm}}{2 k T}\right], \\
\Delta \mathcal{F}_{n}^{ \pm} & =F(n \pm 1 ; \xi)-F(n ; \xi) .
\end{aligned}
$$

Equation (10) is valid for $n=0$ with the boundary condition $R_{0}^{-}=0, P_{-1}=0$. The accessible range of $n$ 's is formally limited upward by the conditions $n \leq N$ and $|x|<1$, either of which may prescribe the maximum value $n_{\max }$, depending on model parameters; at this point, the boundary condition reads $R_{n_{\max }}^{+}=0, P_{n_{\max }+1}=0$.

As seen in Fig. 1, the predictions of this independent chain approximation for the growth of the average helix fraction starting from the zero-helix initial state (blue lines) fit remarkably to those of the full network simulation. We thus conclude that in our system, elastic couplings are completely 
irrelevant. This suggests that the mechanical equilibration following a helix-coil transition affects only very weakly the rates of subsequent events.

To understand why this holds, let us consider an arbitrary event, e.g., $n_{i j} \rightarrow n_{i j}+1$. Immediately after the barrier crossing, the strand tension $t_{i j}=\partial F_{i j} / \partial r_{i j}$ varies by $\delta t_{i j}=\left(\partial t_{i j} / \partial n_{i j}\right) \times 1=\partial^{2} F_{i j} / \partial r_{i j} \partial n_{i j}$. To recover mechanical balance, the network deforms, and the associated strain field decreases (as $1 / r^{2}$ in 2D) with distance from the event. The biases induced by these strains are maximal on the implicated strand itself, which stretches by $\delta r_{i j} \simeq \delta t_{i j} / k_{i j}^{\text {eff }}$, with $k_{i j}^{\text {eff }}$ an effective stiffness; they hence cause at most a relative variation of transition rates: $\delta R_{n_{i j}}^{ \pm} / R_{n_{i j}}^{ \pm}=-\delta\left(\Delta \mathcal{F}_{i j}^{ \pm}\right) / 2 k T$ $\simeq\left(\partial \Delta \mathcal{F}_{i j}^{ \pm} / \partial r_{i j}\right) \times \delta r_{i j} / 2 k T$. Finally, using $\Delta \mathcal{F}_{i j}^{ \pm} \simeq \pm \partial F_{i j} / \partial n_{i j}$, it comes to

$$
\frac{\delta R_{n_{i j}^{ \pm}}^{ \pm}}{R_{n_{i j}}^{ \pm}} \simeq \frac{1}{2 k T} \frac{1}{k_{i j}^{\text {eff }}}\left(\frac{\partial^{2} F_{i j}}{\partial r_{i j} \partial n_{i j}}\right)^{2} .
$$

At the small helix fractions of interest, the order of magnitude for this quantity can be estimated at small stretch ratios so that $w(x) \simeq x^{2}$ [see Eqs. (2) and (3)] and in the limit where all $n_{i j}$ 's vanish. Under these conditions, $k_{i j}^{\text {eff }}$ is the value in an elastic network with identical bonds; for a 2D triangular network, it reads ${ }^{16} k_{i j}^{\text {eff }}=(2 / 3) \partial^{2} F_{i j} / \partial r_{i j}^{2}$. Finally, we obtain for the maximum relative variation of transition rates induced by elastic strains

$$
\eta \equiv \frac{\delta R_{n_{i j}}^{ \pm}}{R_{n_{i j}}^{ \pm}} \sim \frac{a}{\ell_{p} N}\left(\frac{\xi}{N a}-\gamma\right)^{2} .
$$

For our choice of parameters, $\eta \simeq 10^{-5}$, which explains the weakness of elastic coupling effects. In the gels studied in Ref. 1, where $\xi \simeq 30 a$ and $N$ a few hundreds, the quantity $\left(\frac{\xi}{N a}-\gamma\right)^{2} \lesssim 10^{-1}$, which yields an upper bound of at most $10^{-4}$ for $\eta$. This leads us to conclude that elastic couplings are irrelevant to the helix-coil dynamics.

\section{SINGLE CHAIN DYNAMICS AT SMALL HELIX FRACTIONS}

This conclusion suggests that the experimentally observed relaxation dynamics is governed solely by the dynamics of helix-coil transitions for an ensemble on independent and identical chains. We thus focus from now on the model defined in Eqs. (10) and (11). But, anticipating on the coming comparison with experiments, we extend this model to $3 \mathrm{D}$, in which case $w^{\prime}$ is the inverse of the Langevin function, and $w$ can be approximated as ${ }^{17}$

$$
w(x)=\frac{1}{2} x^{2}-\log \left(1-x^{2}\right) .
$$

We choose parameters that correspond to gels with gelatin concentration $c_{\text {gel }}=5$ wt. $\%$ and a shear modulus $G=1400 \mathrm{~Pa}$. For our chemically crosslinked networks, the functionality (the number of strands connected at a node) $f=4$ and the number densities of cross-links $(v)$ and strands $\left(v_{\text {str }}\right)$ are related by $v_{\text {str }}$ $=2 v$. We estimate $v$ using the phantom network expression ${ }^{18}$ for the shear modulus, $G=(f-2) / f \times v k T=v k T / 2$. The number of monomers per strand $N=v_{\text {mon }} / v_{\text {str }}$, with the monomer number density $v_{\text {mon }}=c_{\text {gel }} \rho_{\mathrm{w}} / m_{\text {mon }}$, where $\rho_{\mathrm{w}}$ and $m_{\text {mon }}$ are, respectively, the water mass density and the monomer mass. Taking the average molar mass of residues on gelatin to be 80 Da, ${ }^{19}$ we find $N \simeq 285$.

To estimate the end-to-end distance $\xi$ of a strand, we recall that at small stretch ratios the shear modulus of an elastically isotropic network is very well approximated by $G \simeq-P^{\text {chain }},{ }^{2}$ with $P^{\text {chain }}$ the pressure carried by the network, which can be estimated as $P^{\text {chain }}=-\frac{1}{3 V} \sum_{i<j} r_{i j} \partial F_{i j} / \partial r_{i j} \simeq-\left(v_{\mathrm{str}} / 3\right)$ $\times\left\langle r_{i j} \partial F_{i j} / \partial r_{i j}\right\rangle_{i j}=\xi=-v k T \xi^{2} /\left(N a \ell_{p}\right)$. On this basis, we fix

$$
\xi=\sqrt{\frac{N a \ell_{p}}{2}}
$$

which is of order $31 a$ for $\mathrm{N}=285$ and $\ell_{p}=7 a$.

\section{A. Relaxation of the helix fraction after a quench}

The evolution of the helix fraction as a function of time, after a quench from the high- $T$, zero-helix, state is reported in Fig. 2(a) for the 3D version of our model and various values of $\alpha$, corresponding to equilibrium helix fractions ranging from $3 \%$ to $18 \%$. The $\phi_{h}$ vs $t / \tau_{0}$ curves show the same qualitative behavior as found earlier in 2D: both the equilibrium helix fraction and the relaxation time scale grow with decreasing $\alpha$. The plot in Fig. 2(b) of the departures $\delta \phi_{h}=\phi_{h}^{\mathrm{eq}}-\phi_{h}$ from the thermodynamic equilibrium values $\phi_{h}^{\mathrm{eq}}$ shows that relaxation slows down considerably with decreasing $\alpha$ (i.e., temperature). Moreover, our data feature the same qualitative behavior as observed in stress relaxation experiments, namely,

- as illustrated in Fig. 2(c) for $\alpha=0.07$, all $\delta \phi_{h}$ curves exhibit a final exponential decay $\left[\delta \phi_{h} \sim \exp \left(-t / \tau_{f}\right)\right]$ preceded by a stretched exponential behavior $\left[\delta \phi_{h}\right.$ $\left.\sim \exp \left(-\left(t / \tau_{K}\right)^{\beta}\right)\right]$ with Kohlrausch exponents $<1$ (here, $\beta=0.63 \pm 0.02)$

- the characteristic times $\tau_{f}$ and $\tau_{K}$ grow much more rapidly with decreasing $\alpha$ than the basic time scale $\tau_{0}$ which sets the clock for the activated dynamics [see Fig. 2(d)].

It is striking, moreover, that, since $\tau_{0} \sim 10-20 \mathrm{~s}^{12}$ in the temperature domain studied in Ref. 1 , the relaxation times $\tau_{f}$ and $\tau_{K}$ turn out to lie in ranges that compare quite well with experimental data for $G=1400 \mathrm{~Pa}$. Altogether, our model hence captures all the main features identified from the experimental stress relaxation dynamics.

\section{B. Slowing down mechanism}

The question now is to understand more specifically the nature of the mechanism responsible for the rapid slowing down that occurs when cooling over an $\alpha$ range where equilibrium helix fractions remain quite small.

The reduced strand free-energy $F / k T$ at fixed end-to-end distance $\xi$ [given by Eq. (15)] is plotted in the inset of Fig. 3 for all our $\alpha$ 's. In all cases, $F / k T$ grows monotonously with $n$ hence its minimum value corresponds to the zero helix state: our parameter range lies above (in terms of $\alpha$ and $T$ ) the helix-coil mean-field transition point, where the slope $F^{\prime}(0)=\partial F /\left.\partial n\right|_{n=0}$ vanishes, and its minimum shifts to finite $n$ 's. As the transition point is approached from above, $F^{\prime}(0)$ noticeably decreases, thus causing a broadening of the equilibrium distribution $P^{\mathrm{eq}}$, 


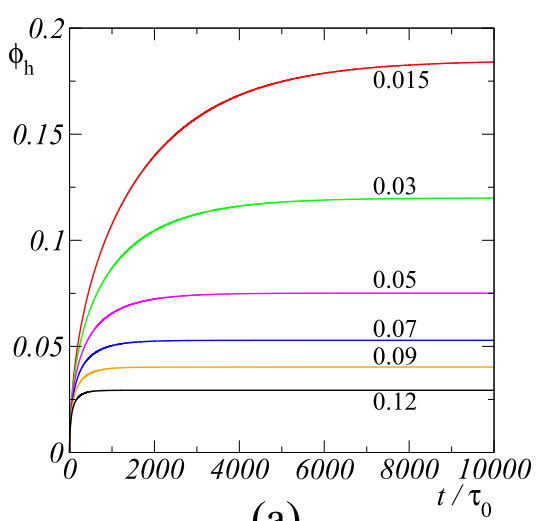

(a)

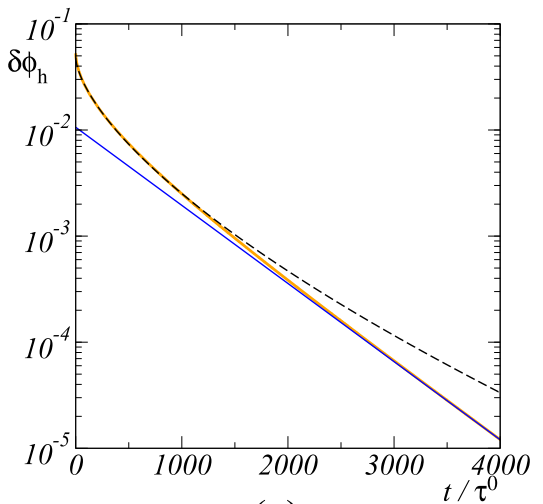

(c)
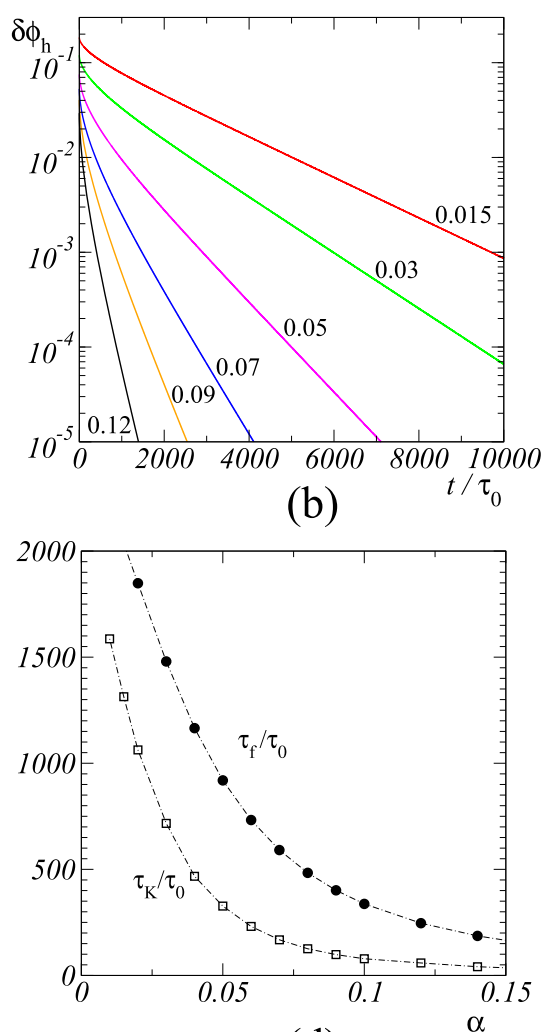

(d)
FIG. 2. Relaxation towards equilibrium in our 3D model of independent chains. (a) The helix fraction $\phi_{h}$ vs $t / \tau_{0}$ for $\alpha=0.015,0.03,0.05,0.07,0.09$, 0.12 , as labeled. (b) The corresponding departures from asymptotic values $\delta \phi_{h}=\phi_{h}^{\mathrm{eq}}-\phi_{h}$ vs $t / \tau_{0}$. (c) $\delta \phi_{h}$ vs $t / \tau_{0}$ for $\alpha=0.07$ (solid orange line) fitted, at large times, by an exponential (solid line: $\sim \exp \left(-t / \tau_{f}\right)$ with $\left.\tau_{f} \simeq 590 \tau_{0}\right)$ and, in the early regime, by a stretched exponential (dashed line $\sim \exp \left(-\left(t / \tau_{K}\right)^{\beta}\right)$ with $\tau_{K} \simeq 190 \tau_{0}$ and $\beta=0.63$ ); the crossover occurs around $\tau_{c o} \simeq 1500 \tau_{0}$. (d) Normalized final relaxation times $\tau_{f} / \tau_{0}$ (full circles) and stretched exponential times $\tau_{K} / \tau_{0}$ (empty squares) vs $\alpha$ (dashed-dotted lines are guides to the eye). which is plotted (left axis, dashed lines) in the main frame of Fig. 3 for our two extremal values of $\alpha$ : 0.12 (black) and 0.015 (red). Slowing down is concomittant with a significant broadening of the equilibrium distribution, i.e., with the growth of pre-transitional fluctuations.

In order to illustrate the sensitivity of the relaxation timescale to the initial slope $F^{\prime}(0)$, we solve analytically in the Appendix the independent chain model within the linear approximation $F=F(0)+k T \lambda n$, where

$$
\lambda=\frac{F^{\prime}(0)}{k T}=\alpha-\alpha_{c}
$$

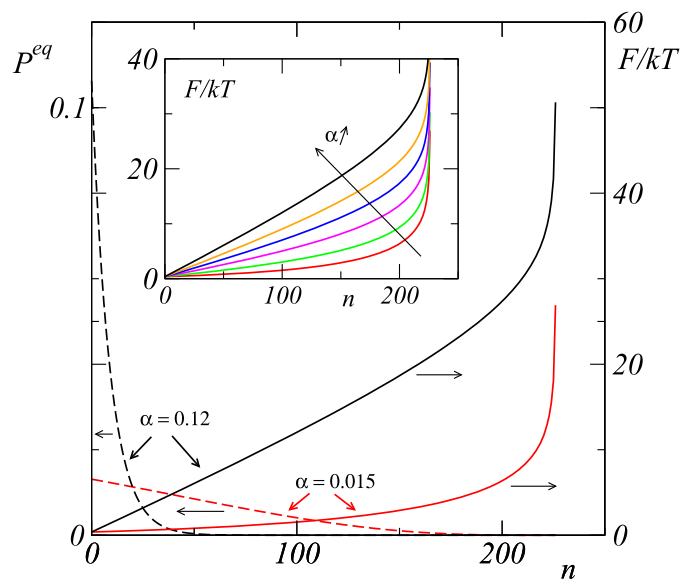

FIG. 3. Inset: $F / k T$ vs $n$ for the same $\alpha$ 's as in Fig. 2(a). Main panel: For our two extremal $\alpha$ values ( 0.015 and 0.12 ), the equilibrium distribution $P^{\text {eq }}$ (dashed lines, left axis) and $F / k T$ (solid lines, right axis) vs $n$. with

$$
\alpha_{c} \equiv \frac{a}{2 \ell_{p}}\left[w\left(x_{0}\right)-\left(x_{0}-\gamma\right) w^{\prime}\left(x_{0}\right)\right]
$$

and $x_{0}=\xi /(a N)$.

We find that the relaxation of the mean helix fraction

$$
\phi_{h}=\sum_{n=0}^{\infty} \frac{n}{N} P_{n}(t)
$$

towards its equilibrium value is given by

$$
\begin{aligned}
\delta \phi_{h} & \equiv \phi_{h}^{\mathrm{eq}}-\phi_{h}(t) \\
& =\frac{1}{2 \pi N} \int_{s_{-}}^{s_{+}} \mathrm{d} \eta \frac{e^{-\eta t}}{\eta^{2}} \sqrt{\left(\eta-s_{-}\right)\left(s_{+}-\eta\right)} .
\end{aligned}
$$

That is, the spectrum of relaxation times extends over the finite interval between $\tau_{\min }=s_{+}^{-1}$ and $\tau_{\max }=s_{-}^{-1}$, with

$$
s_{ \pm}=2 \tau_{0}^{-1}\left[\cosh \left(\frac{\lambda}{2}\right) \pm 1\right] .
$$

It immediately follows that at times $\gg \tau_{\max }$, the asymptotic behavior is exponential, with the characteristic time $\tau_{\max }$. Since the latter identifies with our final time, $\tau_{f}$ therefore reads

$$
\tau_{f}=\frac{\tau_{0}}{2\left(\cosh \left(\frac{\lambda}{2}\right)-1\right)} .
$$

In the small $\lambda$ limit, $\tau_{\min }$ saturates to $\tau_{0} / 4$ while the final time $\tau_{f}$ diverges as

$$
\tau_{f} \sim \frac{4 \tau_{0}}{\lambda^{2}}=\frac{4 \tau_{0}}{\left(\alpha-\alpha_{c}\right)^{2}} .
$$


That is, as $\alpha$ (or temperature) decreases towards the helix-coil transition point of a strand, the broadening of the equilibrium distribution with decreasing $F^{\prime}(0)$ results in the slowing down of relaxation.

In this linear approximation, however, fluctuations are grossly overestimated upon approaching the transition point $\alpha=\alpha_{c}$ : in reality, each strand contains a finite number of monomers, hence the width of the distribution of $n$ cannot grow indefinitely. As is well-known, neither fluctuations nor relaxation time diverge at the nominal transition point in finite size systems.

\section{STRESS RELAXATION}

The previous discussion gives us solid grounds for studying stress relaxation using the independent strand model. As before, strands have the same number of monomers $N=285$ and the length $\xi \simeq 30 a$ fixed by Eq. (15) that corresponds to a $c_{\text {gel }}=5$ wt. $\%$ gel with shear modulus $G=1400 \mathrm{~Pa}$. We monitor the evolution of the macroscopic shear stress after a step strain of amplitude $\varepsilon$ is applied, in the $x z$ plane, to an initially unloaded state. The initial orientation vectors $\underline{r}_{0} / \xi=(\cos \varphi \sin \theta, \sin \varphi \sin \theta, \cos \theta)$ are supposed to be uniformly distributed on the sphere since the unloaded gel is isotropic. In the strained state, strand end-to-end vectors are

$$
\underline{r} \equiv\left(\begin{array}{l}
x \\
y \\
z
\end{array}\right)=\xi\left(\begin{array}{l}
\cos \varphi \sin \theta+\varepsilon \cos \theta \\
\sin \varphi \sin \theta \\
\cos \theta
\end{array}\right)
$$

The time-dependent shear stress reads

$$
\sigma \equiv \sigma_{x z}=v_{\mathrm{str}}\left\langle\frac{\partial F}{\partial r} \frac{x z}{r}\right\rangle
$$

with $v_{\text {str }}$ as the number of strands per unit volume. Since $F / k T$ is fully set by the parameter $\alpha$, the model yields $\sigma /\left(v_{\mathrm{str}} k T\right)$ values, from which stress is obtained using $v_{\text {str }} k T=4 G$, with $G=1400 \mathrm{~Pa}$.

\section{A. Sensitivity of stress response to the initial helix content}

Using this model, we study stress relaxation following two different protocols:
(A) The system, initially unloaded and in the zero-helix state, is simultaneously quenched to the target temperature $T$ and loaded at strain $\varepsilon$.

(B) The unloaded system is initially in equilibrium at the target temperature and then loaded instantaneously at strain $\varepsilon$.

In both cases, loading is applied at $t=0$, with the same strain amplitude $\varepsilon=0.2$ as in the experiments of Ref. 1 .

We report in Fig. 4(a) the subsequent evolutions of $\sigma$ for a temperature corresponding to $\alpha=0.07$. The final stress is naturally the same in both cases since the same equilibrium under strain is reached. The initial stress levels, however, differ conspicuously, which reveals that the initial, zero-helix, state of protocol (A) has a much higher instantaneous elastic modulus than the unloaded equilibrated stated at the target $\alpha$ value, which contains a finite fraction $\phi_{h}^{\mathrm{eq}}=5.3 \%$ of helices. This is in keeping with the analysis of Ref. 9, which shows that the shear modulus of the isotropic system is very well approximated by $G \simeq-P^{\text {chain }}$, where $-P^{\text {chain }}=\left(v_{\text {str }} / 3\right) \times\langle r \partial F / \partial r\rangle$ is the pressure due to chain tension: indeed, so long as $\gamma \mathrm{Na}>r$ - which is the case for gelatin gels in the range of moduli of interest - the chain tension $\partial F / \partial r$, and hence the shear modulus decreases with helix content. Accordingly, while in protocol (B) stress relaxation is only caused by the adaptation of strand helix contents to their strain-induced changes of elongations, the much larger stress drop seen in protocol (A) results predominantly from the strong increase in helix content upon thermal quenching.

We plot in Fig. 4(b) the departure $\Delta \sigma=\sigma-\sigma^{\infty}$ of the stress from its asymptotic value. Both curves present a final exponential decay, with a common relaxation time $\tau_{f} \simeq 596 \tau_{0}$ which, as it should, is for all purposes identical with the value identified from the relaxation dynamics of the unstrained system (see the caption of Fig. 2). In both protocols, the early decay departs from a simple exponential. In protocol (A) — as in experiments — it clearly matches a stretched exponential regime (with $\tau_{K} / \tau_{0} \simeq 690$ and $\beta \simeq 0$.62). In protocol (B), however, the amplitude of this departure is very small, and the stretched exponential fit (with $\tau_{K} / \tau_{0} \simeq 1090$ and $\beta \simeq 0.93$ ) is much less meaningful. The sharp difference in the measured exponents reflects that the distribution of weights of the various modes of the broad relaxation spectrum depends sensitively on the initial state.

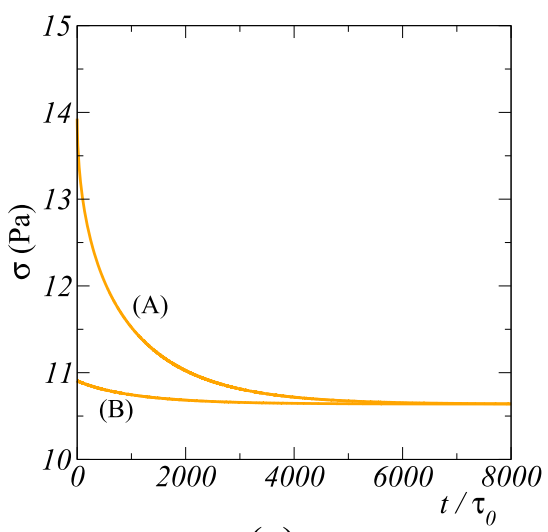

(a)

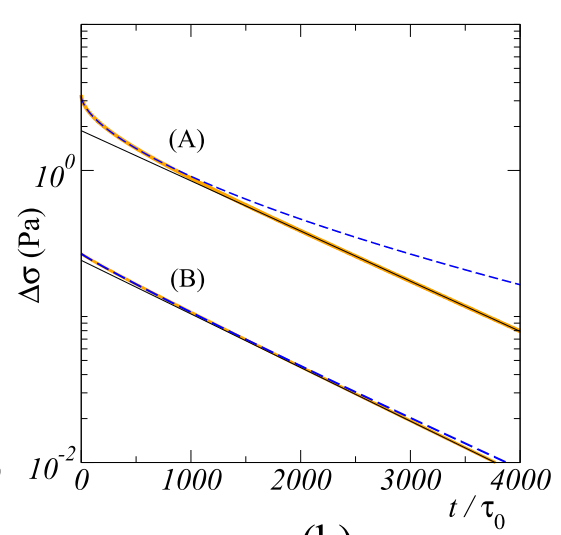

(b)
FIG. 4. Stress relaxation curves in response to a step strain $\varepsilon=0.2$ for protocols (A) and (B), at a temperature corresponding to $\alpha=0.07$. (a): $\sigma$ vs $t / \tau_{0}$. (b): $\Delta \sigma=\sigma-\sigma^{\infty}$ vs $t / \tau_{0}$ (thick orange lines). The thin solid black lines are exponential fits in the terminal regime. The dashed blue lines are stretched exponential fits of the early response. 
TABLE I. For each temperature: measured total stress drop and final relaxation time, and estimated corresponding model parameters (see main text).

\begin{tabular}{lcccc}
\hline \hline & Experimental data & \multicolumn{2}{c}{ Estimated values } \\
\hline$T\left({ }^{\circ} \mathrm{C}\right)$ & $\Delta \sigma(\mathrm{Pa})$ & $\tau_{f}(\mathrm{~s})$ & $\tau_{0}(\mathrm{~s})$ & $\alpha$ \\
\hline 30 & 12.1 & 10500 & 7 & 0.034 \\
32.5 & 4.8 & 4027 & 5.4 & 0.061 \\
35 & 2.35 & 1533 & 4.3 & 0.097 \\
40 & 1.85 & 677 & 2.6 & 0.117 \\
45 & 1.71 & 320 & 1.7 & 0.136 \\
\hline \hline
\end{tabular}

\section{B. Comparison with experiments}

The experiments reported in Ref. 1 used the following protocol: starting from $70^{\circ} \mathrm{C}$, the unloaded sample was quenched to the target temperature $T$ (between 30 and $45^{\circ} \mathrm{C}$ ). The strain step was applied at the end of the thermal transient.

The total duration of the quench and following thermal transient is typically $200 \mathrm{~s}$. On the one hand, this is smaller than all the reported final relaxation times. Hence, in all cases, the initial state was not equilibrium at the target temperature. On the other hand, except at the lowest temperature, this duration is non-negligible compared with the reported relaxation time scales so that protocol (A) (zero helix content when loading is applied) is not experimentally realizable.

In view of the high sensitivity of stress relaxation to initial conditions identified above, a meaningful test of our model can be based only on experimental data obtained from a well-defined initial unloaded state. For this purpose, we have performed a new set of experiments, conducted as described in Ref. 1, except for one difference: we let the system approach as much as possible equilibration at the working temperature before loading. In view of the limits imposed by solvent evaporation and enzymatic degradation ${ }^{1}$ to the full duration of an experimental run, a manageable choice consists in waiting a time $2 \tau_{f}$ after the end of the thermal quench before loading. The data thus obtained for $\tau_{f}$ and the total stress drop $\Delta \sigma$ are reported in columns 2 and 3 of Table I.

The main difficulty we face when proceeding to a test of our predictions against these data is that temperature does not enter the model explicitly but indirectly via the two parameters $\tau_{0}$ and $\alpha$. The former is the Arrhenius time for the controlling

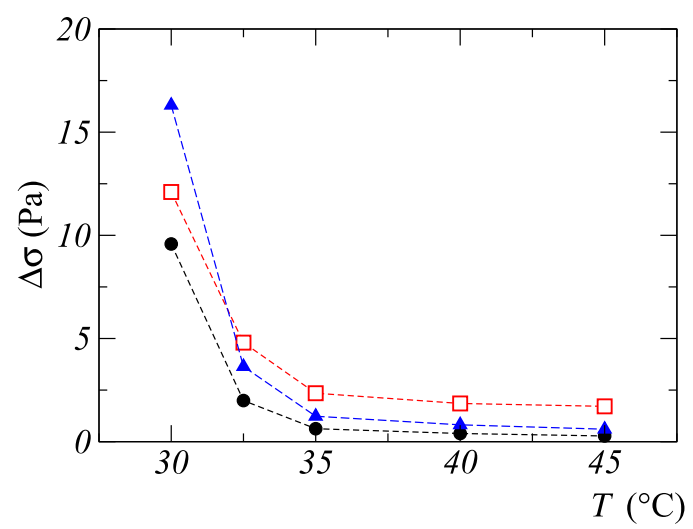

FIG. 6. Comparison of stress drop experimental data (red open squares) with model predictions (full symbols) for initial conditions corresponding to protocol B (equilibrium at working temperature, black circles) and to the partially relaxed state reached at $2 \tau_{f}$ starting from the zero helix state (blue triangles).

cis-trans peptide bond isomerization. We will evaluate $\tau_{0}(T)$ considering that it is $\simeq 12 \mathrm{~s}$ at $25^{\circ} \mathrm{C}$ and that the corresponding activation energy barrier $\simeq 80 \mathrm{~kJ} / \mathrm{mol} .^{11,12}$ The second parameter, $\alpha$, defined in Eq. (9), is a measure of the free-energy difference between the helix and coil states, which vanishes at the helix-coil transition temperature $T_{0}$ of the (infinitely long) polymer chain. About its temperature dependence, we only know that its growth with $T$ is quasi-linear near $T_{0}$, the value of which is unknown. Hence a mapping between $\alpha$ and $T$ has to be inferred from our own data.

This is performed as follows: for a given temperature, using the experimental $\tau_{f}$ and our estimate of $\tau_{0}(T)$, we compute $\tau_{f} / \tau_{0}(T)$; we then interpolate the numerical $\tau_{f} / \tau_{0}$ vs $\alpha$ curve, shown in Fig. 5(a), to infer the $\alpha$ value that corresponds to the chosen temperature (see Table I, last column). Using the curve in Fig. 5(b), we then obtain a predicted value for the stress drop $\Delta \sigma$ at that temperature.

Note that in principle we could as well have used the $\Delta \sigma$ curve to infer the $\alpha$ values and then compare the predicted and measured $\tau_{f} / \tau_{0}(T)$ data. The reason for our choice is that the $\tau_{f}$ value is independent of the initial state, which as discussed above, is difficult to fully control experimentally.

As seen in Fig. 6, the model-predicted $\Delta \sigma$ values compare very favorably with experimental data. Indeed, not only does the model capture the overall shape of $\Delta \sigma(T)$ but, more importantly, the predicted values of the stress drop lie in the

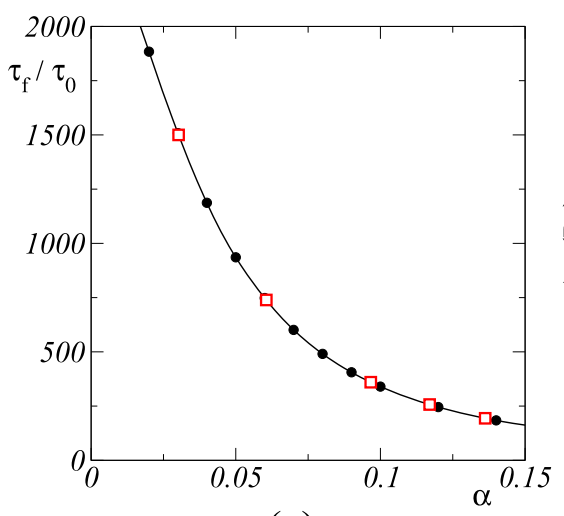

(a)

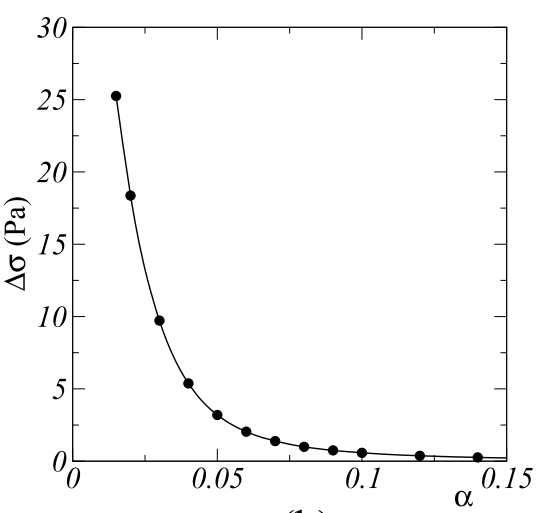

(b)
FIG. 5. Model predictions for the reduced final relaxation time $\tau_{f} / \tau_{0}$ (a) and for the stress drop $\Delta \sigma$ (b). Black circles denote numerical data points, and solid lines show their cubic interpolation. (a): Red squares are the points where the $\tau_{f} / \tau_{0}$ curve achieves the values estimated from experimental data (see main text). The corresponding $\alpha$ values are reported in the inset as a function of $T$. 
correct range. The predicted values, however, are systematically smaller than experimental ones, a discrepancy that might originate, at least in part, from the lack of full equilibration in the experimental initial state. In order to evaluate the importance of this effect, we performed, with the model, stress relaxation tests in which, for each $\alpha$, loading was applied after letting the unloaded system relax from the zero helix state during a time $2 \tau_{f}$, like in experiments. The resulting $\Delta \sigma$ values, reported as triangles in Fig. 6, show that, indeed, at the lower temperatures, the departure from full equilibration induces an increase of $\Delta \sigma$, which turns out to be a sizeable fraction of its value for protocol B. Since the helix content in the partially relaxed states turns out to differ by at most $\lesssim 6 \%$ from its equilibrium value, this observation emphasizes the high sensitivity of stress drop measurements to initial conditions.

\section{CONCLUSION}

In summary, we have here shown that because gelatin is a rather flexible polymer, both collective elastic effects and the disorder in strand contour lengths have a negligible influence on the rates of helix-coil exchange processes. This led us to analyze in detail the effect of helix-coil transition dynamics on stress relaxation in a model of independent strands, which was found to explain the experimentally observed functional form of the relaxation, namely, a stretched exponential followed by a terminal exponential. Moreover, the model predictions concerning both the final relaxation time and total stress drop can be very satisfactorily matched with experimental results.

This supports that despite its schematic character, the model captures the main mechanism that leads to the slow stress relaxation dynamics observed in chemically crosslinked gelatin gels. On this basis, we conclude that the temperature range where glass-like relaxation is experimentally observed lies near but above the helix-coil transition temperature of individual strands and that, in this regime, slowingdown is a signature of the enhancement of helix content fluctuations upon approaching the helix-coil transition of strands.

In a broader perspective, it must be emphasized that the stress relaxation studied here presents empirically the very same behavior as the dielectric response following thermal jumps evidenced by Hecksher et al. ${ }^{5}$ in a set of five organic glasses close below the glass transition temperature. Namely, in both cases, relaxation presents an initial stretchedexponential regime which crosses over to a final exponential decay. In both cases, the transient stretched-exponential decay is a non-linear response effect, which vanishes with the amplitude of jumps, and depends on the distribution of the weights of various relaxation modes in the initial state before quench.

However, the respective physical mechanisms underlying these two situations have hardly anything in common. In our gels, we showed that neither structural disorder (contour lengths) nor long-range couplings are involved in defining the dynamics, which is entirely controlled by processes occurring on well-defined, finite-sized, independent sub-units, which are polymer strands. By contrast, in glasses, while structural disorder is essential, it has never been possible, up to now, to justify a separation into independent sub-units, and it is not known whether terminal exponential relaxation is a general or a specific feature. The comparison between these two situations hence, we think, points to the difficulty of drawing from the empirical similarity of glass-like dynamical responses any direct conclusion about a possible universality of underlying mechanisms.

\section{ACKNOWLEDGMENTS}

We acknowledge stimulating discussions with Tristan Baumberger along the course of this work.

\section{APPENDIX: RELAXATION OF HELIX FRACTION IN THE LINEAR POTENTIAL APPROXIMATION}

In this appendix, we compute the explicit expression for the evolution of the helix fraction in the model defined by linearizing the strand free-energy in $n$ as defined in Eqs. (16) and (17). The rates [Eq. (11)] which then reduce to

$$
R_{n}^{ \pm}=\tau_{0}^{-1} e^{\mp \lambda / 2}
$$

are now $n$-independent. Introducing

$$
R^{+}=\tau_{0}^{-1} e^{-\lambda / 2} \quad \text { and } \quad R^{-}=\tau_{0}^{-1} e^{\lambda / 2},
$$

the reduced problem reads

$$
\begin{aligned}
& \frac{\partial P_{0}}{\partial t}=-R^{+} P_{0}+R^{-} P_{1} \\
& \frac{\partial P_{n}}{\partial t}=R^{+}\left(P_{n-1}-P_{n}\right)+R^{-}\left(P_{n+1}-P_{n}\right) \quad \text { for } n \geq 1 .
\end{aligned}
$$

Its equilibrium distribution can be written as

$$
P_{n}^{\mathrm{eq}}=\left(1-\frac{R^{+}}{R^{-}}\right)\left(\frac{R^{+}}{R^{-}}\right)^{n}
$$

and the equilibrium helix fraction is

$$
\phi_{h}^{\mathrm{eq}}=\frac{1}{N} \frac{R_{+}}{R_{-}-R_{+}} .
$$

To study relaxation following a thermal quench, we solve these equations with the initial condition,

$$
P_{n}(t=0)=\delta_{n 0}
$$

with $\delta$ the Kronecker symbol. The generating function,

$$
\Phi(\varphi, t)=\sum_{n \geq 0} e^{i n \varphi} P_{n}(t),
$$

verifies the initial condition $\Phi(\varphi, t=0)=1$ and obeys

$$
\frac{\partial \Phi}{\partial t}=f(\varphi) \Phi(\varphi, t)+g(\varphi) P_{0}(t)
$$

with

$$
P_{0}(t)=\frac{1}{2 \pi} \int_{0}^{2 \pi} \mathrm{d} \varphi \Phi(\varphi, t)
$$

and

$$
\begin{aligned}
& f(\varphi)=R^{+}\left(e^{i \varphi}-1\right)+R^{-}\left(e^{-i \varphi}-1\right), \\
& g(\varphi)=R^{-}\left(1-e^{-i \varphi}\right)
\end{aligned}
$$


The Laplace transform $\widetilde{\Phi}(\varphi, s)=\int_{0}^{\infty} \mathrm{d} t e^{-s t} \Phi(\varphi, t)$ verifies

$$
\begin{aligned}
s \widetilde{\Phi}(\varphi, s)-\Phi(\varphi, t=0) & =s \widetilde{\Phi}(\varphi, s)-1 \\
& =\widetilde{\Phi}(\varphi, s)+\widetilde{P}_{0}(s) g(\varphi),
\end{aligned}
$$

where

$$
\widetilde{P}_{0}(s)=\frac{1}{2 \pi} \int_{0}^{2 \pi} \mathrm{d} \varphi \widetilde{\Phi}(\varphi, s) .
$$

Equation (A11) solves as

$$
\widetilde{\Phi}(\varphi, s)=\frac{1}{s-f(\varphi)}\left[1+g(\varphi) \widetilde{P}_{0}(s)\right]
$$

which fully determines $\widetilde{\Phi}$ after computing $\widetilde{P}_{0}(s)$ selfconsistently by integration over $\varphi$,

$$
\widetilde{P}_{0}(s)=\frac{I}{1-J}
$$

with

$$
\begin{aligned}
& I=\frac{1}{2 \pi} \int_{0}^{2 \pi} \mathrm{d} \varphi \frac{1}{s-f(\varphi)}, \\
& J=\frac{1}{2 \pi} \int_{0}^{2 \pi} \mathrm{d} \varphi \frac{g(\varphi)}{s-f(\varphi)} .
\end{aligned}
$$

Introducing the notations

$$
s_{ \pm}=R^{+}+R^{-} \pm 2 \tau_{0}^{-1}=2 \tau_{0}^{-1}\left[\cosh \left(\frac{\lambda}{2}\right) \pm 1\right]
$$

and

$$
\Delta(s)=\left(s+s_{+}\right)\left(s+s_{-}\right),
$$

we find

$$
\begin{aligned}
& I=\frac{1}{\sqrt{\Delta}} \\
& J=\frac{1}{2 \sqrt{\Delta}}\left[\sqrt{\Delta}-s+\sqrt{s_{+} s_{-}}\right],
\end{aligned}
$$

where $\sqrt{\Delta}$ is defined to be positive for $s$ real positive.

The mean helix fraction,

$$
\phi_{h}=\sum_{n=0}^{\infty} \frac{n}{N} P_{n}(t)
$$

has the Laplace transform,

$$
\begin{aligned}
\widetilde{\phi}_{h}(s) & =-\left.\frac{i}{N} \frac{\partial \widetilde{\Phi}}{\partial \varphi}(\varphi, s)\right|_{\varphi=0} \\
& =\frac{\sqrt{\Delta}-s-\sqrt{s_{+} s_{-}}}{2 s^{2}} .
\end{aligned}
$$

The singularities of $\widetilde{\phi}_{h}(s)$ in the complex s-plane are

- a simple pole at $s=0$ with residue $\phi_{h}^{\text {eq }}$ [Eq. (A5)],

- a branch cut on the negative real interval $\left[-s_{+},-s_{-}\right]$.
Integrating over the Bromwich contour yields for the inverse Laplace transform,

$$
\phi_{h}(t)=\phi_{h}^{\mathrm{eq}}-\frac{1}{2 \pi N} \int_{s_{-}}^{s_{+}} \mathrm{d} \eta \frac{e^{-\eta t}}{\eta^{2}} \sqrt{\left(\eta-s_{-}\right)\left(s_{+}-\eta\right)} .
$$

The long-time asymptotic behavior is obtained by introducing $\tilde{t}=t s_{-}$and performing the change of variable $z=\eta / s_{-}$ -1 ,

$$
\begin{aligned}
\delta \phi_{h}(t) & =\phi_{h}^{\mathrm{eq}}-\phi_{h}(t) \\
& =\frac{e^{-\tilde{t}}}{2 \pi N} \int_{0}^{\frac{s_{+}}{s_{-}}-1} \mathrm{~d} z e^{-z \tilde{t}} \frac{\sqrt{z\left(\frac{s_{+}}{s_{-}}-1-z\right)}}{(1+z)^{2}} .
\end{aligned}
$$

In the $\tilde{t} \gg 1$ limit, the leading contribution to the integral comes from the immediate vicinity of the $z=0$ lower bound so that

$$
\delta \phi_{h}(t) \simeq \frac{1}{4 \sqrt{\pi} N} \sqrt{\frac{s_{+}}{s_{-}}-1} \frac{e^{-\tilde{t}}}{\tilde{t}^{3 / 2}} .
$$

More precisely, we find the asymptotic behavior

$$
\delta \phi_{h}(t) \simeq \frac{1}{2 \sqrt{\pi} N} \sqrt{\frac{\tau_{f}}{\tau_{0}}}\left(\frac{\tau_{f}}{t}\right)^{3 / 2} e^{-t / \tau_{f}} .
$$

${ }^{1}$ O. Ronsin, C. Caroli, and T. Baumberger, J. Chem. Phys. 144, 064904 (2016).

${ }^{2}$ S. Kutter and E. M. Terentjev, Eur. Phys. J. A 8, 539 (2002).

${ }^{3}$ S. Courty, J. L. Gornall, and E. M. Terentjev, Proc. Natl. Acad. Sci. U. S. A. 102, 13457 (2005).

${ }^{4}$ C. A. Angell, K. L. Ngai, G. B. McKenna, P. F. McMillan, and S. W. Martin, J. Appl. Phys. 88, 3113 (2000).

${ }^{5}$ T. Hecksher, N. B. Olsen, K. Niss, and J. C. Dyre, J. Chem. Phys. 133, 174514 (2010).

${ }^{6}$ R. G. Palmer, D. L. Stein, E. Abrahams, and P. W. Anderson, Phys. Rev. Lett. 53, 958 (1984).

${ }^{7}$ J. T. Bendler and M. F. Shlesinger, J. Mol. Liq. 36, 37 (1987).

${ }^{8}$ J. C. Phillips, Rep. Prog. Phys. 59, 1133 (1996).

${ }^{9}$ C. Caroli and A. Lemaitre, Phys. Rev. E 95, 032501 (2017).

${ }^{10}$ M. Goldstein, J. Chem. Phys. 51, 3728 (1969).

${ }^{11}$ H. P. Bachinger, P. Bruckner, R. Timpl, and J. Engel, Eur. J. Biochem. 90, 605 (1978).

${ }^{12}$ H. P. Bachinger, P. Bruckner, R. Timpl, D. J. Prockop, and J. Engel, Eur. J. Biochem. 106, 619 (1980).

${ }^{13}$ E. Evans and K. Ritchie, Biophys. J. 72, 1541 (1997).

${ }^{14}$ H. B. Bohidar and S. S. Jena, J. Chem. Phys. 100, 6888 (1994).

${ }^{15}$ M. Djabourov, K. Nishinari, and S. Ross-Murphy, Physical Gels from Biological and Synthetic Polymers, 1st ed. (Cambridge University Press, New York, 2013).

${ }^{16}$ S. Feng, M. F. Thorpe, and E. Garboczi, Phys. Rev. B 31, 276 (1985).

${ }^{17}$ A. Cohen, Rheol. Acta 30, 270 (1991).

${ }^{18}$ M. Rubinstein and R. Colby, Polymer Physics (Oxford University Press, New York, 2003).

${ }^{19}$ K. te Nijenhuis, Thermoreversible Networks: Viscoelastic Properties and Structure of Gels, Volume 130 of Advances in Polymer Science (Springer, 1997). 